\title{
Tracheotomy in COVID-19 patients: Optimizing patient selection and identifying prognostic indicators
}

\author{
Thomas James Stubington, $\mathrm{MD}^{1}$ \\ ${ }^{1}$ Royal Derby Hospital
}

May 7, 2020

\begin{abstract}
Background

Tracheotomy, through its ability to wean patients off ventilation, can shorten ICU length of stay and in doing so increase ICU bed capacity, crucial for saving lives during the COVID-19 pandemic. To date, there is a paucity of patient selection criteria and prognosticators to facilitate decision-making and enhance precious ICU capacity.

Methods

Prospective study of COVID-19 patients undergoing tracheotomy ( $\mathrm{n}=12$ ) over a 4-week period (March-April 2020). Association between pre- and post- operative ventilation requirements and outcomes (ICU stay, time to decannulation, and death) were examined.

Results

Patients who sustained $\mathrm{FiO} 2[?] 50 \%$ and PEEP[?] $8 \mathrm{~cm} \mathrm{H} 2 \mathrm{O}$ in the 24h pre-tracheotomy exhibited a favourable outcome. Those whose requirements remained below these thresholds post-tracheotomy could be safely stepped down after $48 \mathrm{~h}$.

Conclusion

Sustained $\mathrm{FiO} 2[?] 50 \%$ and $\mathrm{PEEP}[?] 8 \mathrm{~cm} \mathrm{H} 2 \mathrm{O}$ in the $48 \mathrm{~h}$ post-tracheotomy are strong predictive factors for a good outcome, raising the potential for these patients to be stepped down early, thus increasing ICU capacity.
\end{abstract}

Thomas J. Stubington . 1 , Ali S Mallick, Georgios Garas 3, Emma Stubington . 4, Chetan Reddy . 5, Mohammed S Mansuri . 6

1. MBBS BMedSci MRCS(ENT) PGCert (MedEd), Department of Otorhinolaryngology - Head and Neck Surgery, Royal Derby Hospital, Uttoxeter Road, Derby, DE22 3NE, United Kingdom

2. MBBS MRCS (DOHNS) PhD, Department of Otorhinolaryngology - Head and Neck Surgery, Royal Derby Hospital, Uttoxeter Road, Derby, DE22 3NE, United Kingdom

3. PhD FRCS FEBORL-HNS, Department of Otorhinolaryngology - Head and Neck Surgery, Nottingham University Hospitals NHS Trust, Queens Medical Center Campus, Nottingham, NG7 2UH, United Kingdom

4. BSc, Mres, PhD STOR-I Centre for Doctoral Training, Lancaster university, UK

5. MBBS FANZCA, Department of Anaesthesia, Royal Derby Hospital, Uttoxeter Road, Derby, DE22 $3 \mathrm{NE}$

6. MBChB, Msc, Dip (RMES), FRCS (ORL), Department of Otorhinolaryngology - Head and Neck Surgery, Royal Derby Hospital, Uttoxeter Road, Derby, DE22 3NE, United Kingdom

Corresponding Author: Thomas James Stubington, Dept. Head and Neck Surgery, Royal Derby Hospital, Uttoxeter Road, Derby, DE223NE Thomas.stubington@nhs.net

Short Title 
Prognostic indicators for Tracheotomy in Covid-19 Patients.

Keywords

SARS-CoV-2, Coronavirus, COVID-19, Pandemic, Tracheotomy

Introduction

Coronavirus disease 2019 (COVID-19) has rapidly evolved into a pandemic since the first report emerged from China in December $2019^{1}$. With the number of cases rising globally at an exponential rate and with over $10 \%$ of these requiring Intensive Care Unit (ICU) admission, demand for critical care increasingly threatens to exceed capacity even among the world's most advanced economies ${ }^{2}$. At present, supportive treatment forms the basis of therapy, with trials currently ongoing to unearth the optimal medicinal treatment regimen and vaccine. Tracheotomy, through its ability to wean patients off ventilation, can shorten the ICU length of stay and in doing so increase ICU bed capacity; crucial for saving lives at a population level. ${ }^{3,4}$ Median ICU stay for COVID-19 patients varies widely between countries ranging between 4 to over 20 days. ${ }^{5}$

Tracheotomy constitutes an Aerosol Generating Procedure (AGP), thus potentially exposing the operating surgeon and Operating Room (OR) team to respiratory droplets from the SARS-CoV-2 infected patient ${ }^{6}$. With this added risk in mind it is vital that the potential benefits of a reduced ICU stay associated with performing a tracheotomy are balanced against the risks to healthcare professionals. Despite a number of authors having already published guidelines to minimize risks to healthcare personnel when performing tracheotomy in the COVID-19 positive patient ${ }^{7-11}$, there is currently a paucity of literature on patient selection criteria for this procedure and outcomes data for patients who have undergone tracheotomy in these circumstances. To address this, we present our data from the first 12 COVID-19 patients that underwent tracheotomy in our institution, and propose parameters to inform patient selection by identifying those patients who may be more likely to benefit from the procedure. Furthermore, we discuss potential predictive factors that may help clinicians identify at an early stage (48h post-operatively) those patients who are likely to have a positive outcome post-tracheotomy, which may facilitate decisions to step-down patient care and thus improve the availability of critical care resources to those patients that need it most.

Methods

This was a prospective study of all COVID-19 patients undergoing tracheotomy $(n=12)$ in a Head \& Neck Unit in the United Kingdom during a 4-week period (March-April 2020). Anesthesiological processes and surgical steps pre- and peri-tracheostomy insertion were standardised to minimise risk to staff and improve patient safety during this crucial part of the procedure (see Supplementary Material). Recordings of the patient's Fraction of Inspired Oxygen $\left(\mathrm{FiO}_{2}\right)$ and Peak End Expiratory Pressure (PEEP) were obtained for the 24 hours preceding the procedure, and subsequently collected on a daily basis until the patient was either decannulated and discharged from hospital, or died. Fluctuations in these values, which occurred due to patient intervention/movement were removed in order to facilitate calculation of representative averages for these values. The number of days that patients were kept under sedation and number of days taken for decannulation were also recorded.

Following our experience with our first 5 tracheotomies and in accordance with our local protocol (see Supplementary Material) and published literature ${ }^{7-9}$, we instituted selection criteria for all subsequent tracheotomies as follows:

- Patients should ideally be at least 14 days post-positive swab result

- Low oxygen requirements $\left(\mathrm{FiO}_{2}[?] 40 \%\right)$, sustained for at least 24 hours

- Patient able to tolerate clamped tube for 1 min in ICU ('clamp test')

- Two failed trials of sedation withholding prior to considering tracheotomy

- Patients that will not require prone ventilation

Correlation between data sets was determined using the 'R' statistical software (v3.6.1, (C) The R Foundation, 
Vienna, Austria). Data were ranked, and Spearman's rank correlation coefficient was calculated to determine association between data sets.

\section{Results}

In total, 12 COVID-19 patients underwent surgical tracheotomy over a 4-week period (March-April 2020), of which two died (patients $\mathrm{B}$ and $\mathrm{C}$ ). Patient $\mathrm{B}$ had $\mathrm{FiO}_{2}$ values ranging between $81-90 \%$ in the 24 hours before tracheotomy, whilst patient $\mathrm{C}$ had their tracheotomy performed 4 days after having a positive swab result. Of the remaining patients that survived, patients $\mathrm{D}$ and $\mathrm{E}$ took the longest to be decannulated, both of whom had pre-operative $\mathrm{FiO}_{2}$ values [?]50\%, as well as high PEEP values (Table 1).

The data suggest that a patient's pre-operative status in the 24 hours preceding the procedure may highlight those patients likely to benefit from tracheotomy. Figure 1 illustrates each patient's pre-operative $\mathrm{FiO}_{2}$ concentration and PEEP requirements in the format of a bubble plot, which suggests that an $\mathrm{FiO}_{2}$ of [?]50\% with a PEEP of [?] $8 \mathrm{~cm}$ of $\mathrm{H}_{2} \mathrm{O}$ in the 24 hours preceding tracheotomy may be associated with a worse outcome, as the patients that did not fulfil these criteria in the 24 hours preceding tracheotomy either died or had a prolonged wean of ventilation despite the procedure.

Cumulative data suggested that patients requiring an $\mathrm{FiO}_{2}$ of [?] $50 \%$ and $\mathrm{PEEP}$ of [?] $8 \mathrm{~cm}$ of $\mathrm{H}_{2} \mathrm{O}$ in the first 48 hours following tracheotomy tended to have a more favorable outcome compared to those exceeding these values. To examine this in greater depth, the proportion of time for each patient at which the PEEP was [?] $8 \mathrm{~cm} \mathrm{H} \mathrm{H}_{2} \mathrm{O}$ and the proportion of time $\mathrm{FiO}_{2}$ was [?]50\% across all days were calculated regardless of length of follow-up. From this, the average proportion of time that PEEP was [?] $8 \mathrm{~cm} \mathrm{H}_{2} 0$ and average proportion of time that $\mathrm{FiO}_{2}[?] 50 \%$ were calculated, with the patients subsequently ranked accordingly. These steps were then repeated but with only looking at the data for up to day 2 post-tracheotomy to look for early prognostic indicators (see Supplementary Figures 1 and 2). The ranks for both parameters were then plotted on a scatter plot (Figure 2), which illustrates that rank at day 2 post-tracheotomy strongly correlates with rank from all days, and therefore with the patient's final outcome $(\rho=0.955, \mathrm{P}<0.05)$.

\section{Discussion}

This is the first report of patient outcomes following tracheotomy in COVID-19 patients, and on the basis of our institutional experience we propose criteria that offer a pragmatic solution to facilitate patient care whilst minimizing risks to healthcare workers.

Given the risk that tracheotomy in COVID-19 patients poses to healthcare workers through aerosolization of SARS-CoV-2 virions, it is paramount that these risks are carefully balanced against potential benefits to patient care. Concerns around infection in COVID-19 patients stem from previous experience from SARS$\mathrm{CoV}$, which posed a particular risk to healthcare workers as peak viral load tended to occur 7-10 days post-infection ${ }^{10}$. In contrast, COVID-19 patients appear to have the highest viral load at the onset of infection and this subsequently declines over time, which may account for the speed at which this novel coronavirus is spreading within the community ${ }^{11}$. Furthermore, although it has been shown that SARSCoV-2 RNA can be detected in patients up to 20 days or longer post-infection, it is unclear whether this represents patients shedding live virus, or if this reflects shedded virions inactivated by host antibodies ${ }^{11}$. In the context of planning a tracheotomy, this has two important implications; first, a positive test does not reflect the degree of infectivity of a patient, and second, on the basis of current data we can draw a cautious degree of reassurance that patients are likely to be less infectious the further away they are from their initial presentation. Thus, we believe that undertaking a tracheotomy at least 14 days following a positive swab result, in conjunction with wearing full Personal Protective Equipment (PPE) and taking all the necessary steps to minimize aerosolization peri-tracheotomy insertion (see Supplementary Material) presents a pragmatic solution to minimizing risk to staff. To date, none of the OR staff who have been involved with undertaking tracheotomies in COVID-19 patients has tested positive for the disease in our institution. 
In light of the relative uncertainties that exist in the treatment of patients with COVID-19, patient selection for tracheotomy will ultimately be refined through experience. The selection criteria proposed in this study $\left(\mathrm{FiO}_{2}[?] 50 \%\right.$ and $\mathrm{PEEP}[?] 8 \mathrm{~cm}$ of $\mathrm{H}_{2} \mathrm{O}$ in the 24 hours prior to the tracheotomy) were derived on the basis of our early experience with the first five COVID-19 tracheotomies; of which, one survived (patient A), two died (patients B and C) and the remaining two had a prolonged wean off the ventilator (patient D, who was taken off ventilation at day 17 post-procedure, and patient $\mathrm{E}$, who continues to be on ventilation at the time of writing). Of the 4 patients who had less favorable outcomes, three had either an $\mathrm{FiO}_{2}[?] 50 \%$ or a PEEP requirement exceeding $8 \mathrm{~cm} \mathrm{H}_{2} \mathrm{O}$. The 'anomaly' was patient $\mathrm{C}$ who despite being within an acceptable range for both $\mathrm{FiO}_{2}$ and PEEP unfortunately died. In retrospect, this patient probably had their tracheostomy too early at day 4 post-intubation; it is thus vital that ICU teams liaise closely with surgical teams when identifying potential candidates for tracheotomy, and put forward those patients who have demonstrated improvement in their clinical course.

From this study, two important findings emerge in terms of the prognostic value of ventilation-related parameters prior to tracheotomy. These are an $\mathrm{FiO}_{2}$ requirement [?]50\% and $\mathrm{PEEP}[?] 8 \mathrm{~cm} \mathrm{H}_{2} \mathrm{O}$ in the 24 hours prior to tracheotomy with all patients exceeding these cut off values either experiencing a prolonged wean and dying or failing to improve and continuing to require ICU support. Satisfying both the $\mathrm{FiO}_{2}$ and PEEP criteria is equally important when considering tracheotomy in COVID-19 patients failing trial(s) of extubation. The case of patient $\mathrm{D}$ illustrates why consideration of $\mathrm{FiO}_{2}$ requirements alone is not adequate when it comes to COVID-19 patients. Even if pre-operative $\mathrm{FiO}_{2}$ requirements remain below the $50 \%$ cut off, COVID-19 patients are unlikely to do well following tracheotomy if their PEEP requirements exceed $8 \mathrm{~cm} \mathrm{H}_{2} \mathrm{O}$. The reason is that the high PEEP dependence makes them less able to tolerate the combination of a reduction in $\mathrm{FiO}_{2}$ to $21 \%$ during tracheal exposure and the subsequent cessation of ventilation prior to tube exchange, both key recommendation for tracheotomy in COVID-19 patients. ${ }^{12}$

Furthermore, we have demonstrated that there is a correlation $(\rho=0.955, p<0.05)$ between the proportion of time patients' PEEP values are [?] $8 \mathrm{~cm} \mathrm{H}_{2} \mathrm{O}$ and their $\mathrm{FiO}_{2}$ values are [?] $50 \%$ in the first 48 hours following tracheotomy with the respective values (ventilation requirements) across all days of their stay in critical care. This is of clinical importance because it could potentially permit prognostication through early riskstratification; with those patients whose ventilator requirements consistently remain below the described cut off values (i.e. $\mathrm{FiO}_{2}[?] 50 \%$ and $\mathrm{PEEP}[?] 8 \mathrm{~cm}$ of $\mathrm{H}_{2} \mathrm{O}$ ) for the first 2 post-operative days to represent a subgroup that can potentially be safely stepped down to another clinical area outside the ICU environment at that stage. This has the potential of freeing up intensive care beds as early as day 2 post-tracheotomy for other patients that need it more. Furthermore, with the emergence of field hospitals to help cope with the increased patient demand from the COVID-19 pandemic, this could assist in identifying those patients who following tracheotomy are suitable for transfer to such facilities, further enhancing ICU capacity, a precious and limited resource in the fight against COVID-19.

Prior to concluding, it is important to consider the strengths and limitations of this study. The key limitation relates to the small number of patients $(n=12)$. Another relates to the novelty of the disease studied meaning that it is likely that further refinements to the proposed criteria will be needed in the future as our understanding of the pathophysiology of COVID-19 evolves. Finally, only two prognostic parameters were studied. Despite these limitations, this study also features a number of key strengths. It is the first to look into the development of formal selection criteria for tracheotomy in ventilated patients with COVID19 and the first to determine early prognostic factors for these patients. Moreover, this has been done in a quantitative manner. By providing actual cut off values for pre- and post-tracheotomy ventilatory requirements, it facilitates patient selection, permits risk stratification and in doing so can directly assist clinical decision-making and inform policy.

In conclusion, this study presents for the first time measurable patient selection criteria for tracheotomy in COVID-19 patients, illustrating that an $\mathrm{FiO}_{2}[?] 50 \%$ and PEEP[?] $8 \mathrm{~cm} \mathrm{H}_{2} 0$ in the 24 hours prior to tracheotomy are useful markers in helping to identify those patients that are most likely to benefit from a tracheotomy. It has also shown that patients that are able to remain below these threshold values in the first 
48 hours following tracheotomy are likely to exhibit a favourable outcome and can thus be stepped down from an intensive care setting at that (early) stage, freeing up vital capacity for other critically ill COVID-19 patients in need of urgent ICU care.

Bibliography

1. Chan JFW, Yuan S, Kok KH, et al. A familial cluster of pneumonia associated with the 2019 novel coronavirus indicating person-to-person transmission: a study of a family cluster. Lancet . 2020;395(10223):514523.

2. Remuzzi A, Remuzzi G. COVID-19 and Italy: what next? Lancet . 2020;395(10231):1225-1228.

3. Griffiths J, Barber VS, Morgan L, Young JD. Systematic review and meta-analysis of studies of the timing of tracheostomy in adult patients undergoing artificial ventilation. Br Med J . 2005;330(7502):1243-1246.

4. Andriolo BN, Andriolo RB, Saconato H, Atallah ÁN, Valente O. Early versus late tracheostomy for critically ill patients. Cochrane Database Syst Rev . 2015;2017(6).

5. ICNARC. Intensive Care National Audit and Research Centre ICNARC Report on COVID 19 in Critical Care .; 2020. file://C:/Users/user/Downloads/ICNARC COVID-19 report.pdf.pdf.

6. Tran K, Cimon K, Severn M, Pessoa-Silva CL, Conly J. Aerosol generating procedures and risk of transmission of acute respiratory infections to healthcare workers: A systematic review. PLoS One . 2012;7(4).

7. Heyd CP, Desiato VM, Nguyen SA, et al. Tracheostomy Protocols during COVID-19 Pandemic. Head Neck . April 2020:hed.26192.

8. Skoog H, Withrow K, Jeyarajan H, et al. Tracheotomy in the SARS-CoV-2 pandemic. Head Neck. April 2020:hed.26214.

9. David AP, Russell MD, El-Sayed IH, Russell MS. Tracheostomy guidelines developed at a large academic medical center during the COVID-19 pandemic. Head Neck. April 2020:hed.26191.

10. Peiris JSM, Chu CM, Cheng VCC, et al. Clinical progression and viral load in a community outbreak of coronavirus-associated SARS pneumonia: A prospective study. Lancet . 2003;361(9371):1767-1772.

11. To KK-W, Tsang OT-Y, Leung W-S, et al. Temporal profiles of viral load in posterior oropharyngeal saliva samples and serum antibody responses during infection by SARS-CoV-2: an observational cohort study.Lancet Infect Dis . 2020;0(0).

12. Pichi B, Mazzola F, Bonsembiante A, et al. CORONA-steps for tracheotomy in COVID-19 patients: a staff-safe method for airway management. Oral Oncol . 2020:104682.

\section{Hosted file}

Tracheostomy_in_COVID-19_Table_1.docx available at https://authorea.com/users/312328/ articles/449114-tracheotomy-in-covid-19-patients-optimizing-patient-selection-andidentifying-prognostic-indicators

\section{Hosted file}

Tracheostomy_of_COVID-19_Fig1.docx available at https://authorea.com/users/312328/articles/ 449114-tracheotomy-in-covid-19-patients-optimizing-patient-selection-and-identifyingprognostic-indicators

\section{Hosted file}

Tracheostomy_in_COVID-19_Fig2.docx available at https://authorea.com/users/312328/articles/ 449114-tracheotomy-in-covid-19-patients-optimizing-patient-selection-and-identifyingprognostic-indicators 


\section{Hosted file}

Tracheostomy_in_COVID-19_Supplementary_Table_1.docx available at https://authorea.com/users/ 312328/articles/449114-tracheotomy-in-covid-19-patients-optimizing-patient-selectionand-identifying-prognostic-indicators

\section{Hosted file}

Tracheostomy_in_COVID-19_Supplementary_table_2.docx available at https://authorea.com/users/ 312328/articles/449114-tracheotomy-in-covid-19-patients-optimizing-patient-selectionand-identifying-prognostic-indicators

\section{Hosted file}

RDH_TRACHEOSTOMY_PROTOCOL_COVID.docx available at https://authorea.com/users/312328/articles/ 449114-tracheotomy-in-covid-19-patients-optimizing-patient-selection-and-identifyingprognostic-indicators 